Postprint: Please cite:

Isobel Falconer (1999), 'J J Thomson and the discovery of the electron' Physics Education, vol 32, pp226-231, http://iopscience.iop.org/0031-9120/32/4/015

\title{
J J Thomson and the discovery of the electron
}

\author{
Isobel Falconer St Andrews, UK
}

One experiment, more than any other, is often associated with the 'discovery of the electron' in 1897. This is J J Thomson's determination of the mass to charge ratio $(\mathrm{m} / \mathrm{e})$ of cathode rays by deflecting them in magnetic and electric fields. Yet this experiment was performed two months after Thomson first announced that cathode rays were very small, negatively charged particles. So why was it important? I look at Thomson's route to, and conduct of, the experiment, and then at how his ideas were received.

Born in 1856, Joseph John Thomson was the son of a Manchester bookseller. He was educated at Owens College, Manchester, and Trinity College, Cambridge. In 1884, after four years of research, mainly theoretical, he was elected Cavendish Professor of Experimental Physics at Cambridge at the early age of 28.

Upon his election Thomson began experiments on gaseous discharge. He saw this as a way of untangling the relationship between the ether and chemical atoms. In 1896, with his student Ernest Rutherford, he achieved his most outstanding success to date. Working with the newly discovered x-rays, they established the theory that electric conduction through gases took place by splitting the gas molecules into oppositely charged ions.

\section{Cathode rays}

Thomson then began to assimilate other phenomena into his newly successful theory. Cathode rays were an obvious target. They had just sprung into prominence as the origin of $\mathrm{x}$-rays, produced when cathode rays hit a target. Cathode rays were discovered in 1857 by Julius Plücker. They are observed at very low pressures in an electric discharge tube. Although invisible, they cause fluorescence where they hit the wall of the tube opposite the cathode.

By 1896 it was known that they could be deflected in a magnetic field, and that they cast strong shadows, suggesting that they travelled in straight lines from the cathode. In 1893 Heinrich Hertz had shown that they could pass through thin metallic films. This topic was pursued by his student Philipp Lenard. Such rays became known as Lenard rays. In 1895 Jean Perrin had demonstrated that the rays carried an electric charge. 
With the discovery of x-rays, much discussion was suddenly focused upon cathode rays. Two views of their nature came into open conflict. One was that they were negatively charged particles, probably atoms. The other was that cathode rays were a phenomenon of the ether, akin to light. In late 1896 Thomson turned his attention to cathode rays. He examined their magnetic deflection, and then modified Perrin's experiment to show more conclusively that an electric charge was an indispensable property of the rays. He also considered Lenard's results on the properties and absorption of Lenard rays.

On 30 April 1897, at a Royal Institution Friday Evening Discourse, Thomson announced his conclusion that cathode rays are small negatively charged particles which are a universal constituent of atoms. He supported his suggestion by the results of his first m/e experiment, which relied on the heating effect of the rays. His results gave a mass to charge ratio about 1000 times smaller than that for the hydrogen ion, hitherto the smallest known. He called the particles 'corpuscles', but they have since become known as 'electrons', and Thomson has been hailed as their discoverer.

\section{The m/e experiment}

The experiment upon which this article focuses was Thomson's second method of determining the mass to charge ratio of the corpuscle. He performed it in late June or July 1897, two months after his first suggestion that cathode rays were corpuscles.

The experiment relied on electrostatic deflection of cathode rays. Hertz's inability, 13 years previously, to show an electrostatic deflection had been held to support the ether view of the rays. Cathode rays were produced at the cathode, on the left of the apparatus (figure 1), and travelled to the right, passing through the anode which acted as a collimating tube. They entered a region where either electric or magnetic deflection fields might be applied, and then hit the end of the tube, causing fluorescence. Deflection measurements, made in the dark, were obtained by moving a luminous needle with a screw over the measuring scale until it coincided with the fluorescent patch.

The electrostatic deflection was given by

$$
\Theta=\mathrm{Fel} / \mathrm{m} v^{2}
$$

$(\Theta=$ electric angular deflection, $F=$ applied electric intensity, $\mathrm{l}=$ length of electric plates). If a magnetic force was applied, extending over the same area as the electric plates, then

$$
\Phi=H E l / m v
$$

( $\Phi=$ magnetic angular deflection, $H=$ applied magnetic field intensity). If the magnetic field was varied until the magnetic deflection was the same as the electric deflection, the equations simplified to

$$
\mathrm{Fel} / \mathrm{mv}^{2}=\mathrm{Hel} / \mathrm{mv} \text { or } v=\mathrm{F} / \mathrm{H}
$$




$$
m / e=H^{2} l / F \Phi
$$

$\Theta$ was measured by measuring the electric deflection alone.

Thomson made measurements on air, hydrogen and carbonic acid gas, and tried both aluminium and platinum electrodes. His results for $m / e$ varied from $1.1 \times 10^{-7}$ to $1.5 \times 10^{-7}$ grams per coulomb, and were in general agreement with his earlier results.

He published his results in October 1897. He reiterated his conclusion that the cathode rays were very small charged particles which were the constituents of atoms, and he proposed an atomic model based on them.

\section{Thomson's route to the m/e experiment}

In identifying cathode rays as corpuscles, Thomson adhered to the particle view of cathode rays, ostensibly because this was definite and its consequences could be predicted, whereas we were ignorant of the laws governing the ether (Thomson 1897b, p293). His attitude was symptomatic of the Mechanical Philosophy through which many British physicists sought a unified theory of nature. The Mechanical Philosophy was the belief that all phenomena could be described ultimately in terms of matter in motion. Thus, in investigating an unknown phenomenon such as cathode rays, Thomson's first interest was in their velocity and mass.

Earlier, during the 1880s, Thomson had pursued the Mechanical Philosophy to its mathematical conclusion. His results led him to believe that 'a theory of matter is a policy rather than a creed. Its object is to connect or coordinate apparently diverse phenomena, and above all to suggest, stimulate and direct experiment' (Thomson 1907, p1). Thomson manipulated his theories in just this way. He seldom allowed them to be tied closely to experimental 'facts' which might limit their scope for imaginative extension to other phenomena or further experiment.

His cathode ray work shows this clearly. His corpuscle hypothesis seems based on only two experimental results. First, experiments by Lenard and by himself showed that the magnetic deflection of cathode rays was independent of the electrodes or gas through which they passed. This suggested that the particles were the same in all cases. Second, Lenard had shown that Lenard rays travelled much further through a gas than one would expect for an atomic sized particle. Furthermore, their absorption was inversely proportional to the density of the gas. This suggested that the particles might be very small and be interacting with individual constituents of the gas molecules.

Rather than introduce two new particles, Thomson characteristically made one do both jobs. The cathode rays, he said, were small corpuscles which were themselves the building blocks of atoms. He had been playing with ideas of structured and/or divisible atoms for the previous 15 years, so this explanation 
in 1897 is hardly surprising. It is typical of Thomson's attempts to unify physics by seeking theories which would explain as many different phenomena as possible.

On 30 April 1897 Thomson's theory met with general scepticism. Even George FitzGerald, a most sympathetic listener, considered that he had gone much further than his experimental data warranted. FitzGerald made the alternative suggestion that the cathode ray particles were free electrons, as provided for by the theory of Joseph Larmor. Such electrons were supposed to be centres of strain in the ether. They explained discrete electric charges, but were independent of matter. Thus FitzGerald accepted the particulate nature of cathode rays. But he dissented from the idea that these same particles might make up atoms. In Thomson's terms he was introducing an unnecessary extra hypothesis.

By July 1897 Thomson had several reasons for his m/e experiment. He had extended the corpuscle theory in many directions: he explored its implications for ionization and discharge, he devised atomic models, and he speculated about the structure of molecules. His aim was to extend the scope of corpuscles and demonstrate (contrary to FitzGerald) that they were constituents of atoms. He noted that the specific inductive capacities of gases were approximately additive, implying that the electrical moment of each atom was very high. This led him to suggest that the small value of $\mathrm{m} / \mathrm{e}$ might be due to a large charge as well as a small mass. These speculations pre-dated his attempts to deflect cathode rays electrostatically and provide a motive for them.

\section{Obtaining an electrostatic deflection}

Initially Thomson did not invest much effort in the attempt to deflect cathode rays electrically, he merely cannibalized the apparatus previously used to measure the magnetic spectrum of the rays. He found no deflection. But he noticed that when the rays were on, a discharge passed readily between the two deflecting plates, indicating that the cathode rays turned the gas into a conductor. He realized that the conducting gas screened out the applied field.

The secret to obtaining an electrostatic deflection was clearly to get rid of the residual gas in the tube. The difficulty was to obtain a low enough pressure to prevent ionization and conduction, and yet to initiate a discharge.

One source of residual gas was mercury vapour from the pump itself. Another potential source of vapour was the pressure gauge, and Thomson seldom used one, recording his pressures simply as 'low', 'very low' etc, judging them by the behaviour of the discharge tube.

By 1897 the pump generally used in the Cavendish was a Topler mercury pump worked by hand. It had to be worked for half a day before a good cathode 
ray vacuum was obtained. Before Thomson managed to observe an electric deflection of the rays his assistant Ebeneezer Everett ran the tube for several days pumping all the time.

The purpose of running the discharge tube while pumping was to get rid of all the gas adsorbed on the walls and electrodes. Otherwise this was released throughout the experiment and destroyed the vacuum. 'Baking' the discharge tube was becoming a recognized technique for getting rid of this adsorbed gas, but Thomson's attitude was inconsistent. He used it only when the necessity for doing so was forcibly brought to his attention.

Having attained very low pressure, a further problem arose: below a certain critical pressure the potential needed for discharge rose rapidly and discharge soon became impossible because the discharge tubes broke. In 1883 De la Rue and Muller found that increasing the diameter of the discharge tube lowered the critical pressure. Thomson appears to have designed his cathode ray tube of 1897 with this in mind — the bulb is about $10 \mathrm{~cm}$ wide around the cathode before narrowing to collimate the beam. Hertz, working 13 years earlier, was unaware of De la Rue and Muller's results. His discharge tube was a uniform width of about $2.5 \mathrm{~cm}$ as shown in figure 3. Had he attained a low enough pressure to observe electrostatic deflection, he may not have been able to obtain a discharge.

\section{Results of the experiment}

Eventually, then, Thomson observed the electrostatic deflection he had been seeking. He was now able to measure m/e by his second method, as outlined above. Thomson judged that, "This method of determining the values of $\mathrm{m} / \mathrm{e}$ is much less laborious and probably more accurate than the former method' (Thomson 1897b, p310) and the physics community has concurred with his judgement. Nevertheless, his experiment was far from accurate.

Thomson discussed only two sources of error in his experiment. First, the magnetic force was assumed to be confined to the space between the electric plates, which was only approximately true. This was a systematic error which increased the measurement of m/e. He made no attempt to assess the increase.

Secondly, under either deflection, the cathode rays spread out into a spectrum. Rather than a bright fluorescent spot on the end of his tube, Thomson was observing a patch several millimetres long, introducing an error of up to $20 \%$. The significance of this depends on whether he measured the electrostatic and magnetic deflections one after the other, or whether he opposed them to get a null deflection, an inherently more accurate method. Accounts written much later state that he opposed the forces, and this was certainly a subsequent refinement of the method. But there is nothing in the 1897 paper to suggest that he did so - the implication is that he did not.

For Thomson's purposes, attempts at precision were a waste of time: in October 1897, having reiterated his corpuscle suggestion, he plunged into speculations 
on atomic structure, viewing the atom as an aggregation of corpuscles. The theory was too complex to allow more than a qualitative discussion. Thomson was confident of his m/e results to within an order of magnitude or so, and this he considered good enough.

\section{Corpuscles to electrons}

Other physicists, however, did not agree. The suggestion that atoms might be composed of corpuscles smacked too much of alchemy to be readily accepted and Thomson's experiments were not sufficiently definite to establish this. The main reason why the results of the $\mathrm{m} / \mathrm{e}$ experiment rapidly became important was that they also supported FitzGerald's alternative electron suggestion.

In this form, that cathode rays were 'electrons', independent of matter, Thomson's work was accepted rapidly, especially after his experiments of 1899 which showed that the charge was equal to the unit of electrolytic charge. H A Lorentz, whose electron theory was similar to Larmor's and was far more influential on the Continent, seized upon the electron interpretation and incorporated it into his theory. Moreover, a 'free electron' was some sort of structure in the ether. Hence this suggestion was acceptable even to the protagonists of the ether view of cathode rays. By 1900 the cathode ray controversy had virtually died out.

By the time it was realized that Thomson was right, and that cathode ray particles were an essential part of atomic structure, the word 'electron' was inextricably associated with the particles. Thomson's term 'corpuscle' was forgotten, but it contributed an added meaning to 'electron', that of being an elementary particle, the first to be discovered, and a fundamental constituent of atoms.

This realization waited upon three developments in physics. First came appreciation of the enormous theoretical possibilities if matter was composed of electrons. The mass of Lorentz and Larmor's electrons was electrical in origin (an idea first suggested by Thomson in 1881). If these electrons were also the fundamental particles from which atoms were made, then the entire mass of the universe might be electrical. This idea promised a great advance in the search for a unified theory of physics and was extensively developed in the early twentieth century.

Experimentally, Thomson's work, and the new electron ideas, were confirmed by many others. Most notable was Walter Kaufmann, a highly skilled experimentalist. Several people, including the Curies, nominated Kaufmann jointly with Thomson for the Nobel Prize, reasoning that Thomson's theories would not have been accepted without Kaufmann's supporting evidence.

The third development was also largely experimental: the discovery and investigation of radioactivity. By the early 1900s radioactivity was providing ample evidence that atoms could and did split up and change their chemical nature. Thomson's atomic model was the only one which 
gave an explanation for this. Moreover, measurements of m/e for beta rays showed them to be the same as cathode rays.

\section{The importance of the $m / e$ experiment}

For Thomson, his cathode ray work was one step along the way to establishing a coherent theory of gaseous discharge. It was for this, rather than the cathode ray work, that he won the Nobel Prize in 1906. Neither cathode rays, corpuscles nor electrons were mentioned in the citation. Indeed, for many at the time, Thomson was not the clear-cut 'discoverer of the electron'. Alternative accounts, in which Thomson was of only minor importance, viewed developments by Lorentz, Larmor, Zeeman or Wiechert as the significant steps which established the existence of electrons (e.g. Kaufmann 1901).

Viewed with hindsight, though, it was Thomson who made the nineteenth century electron 'real'. Arriving at the theoretical idea of an electron was not much of a problem in 1897. But Thomson pinpointed an experimental phenomenon in which the electron could be identified, manipulated and experimented upon. He did this most clearly in the m/e experiment, showing how electrons could be deflected magnetically and electrically, how measurements could be made upon them, and how to attach meaning to those measurements. Through the m/e experiment electron theory changed from an abstract mathematical hypothesis to an empirical reality, expanding its meaning in the process.

\section{References and further reading}

Davis E A and Falconer I $1997 \mathrm{JJ}$ Thomson and the Discovery of the Electron (London: Taylor and Francis)

Falconer I 1987 Corpuscles, electrons and cathode Rays: J J Thomson and the 'Discovery of the Electron' Br. J. Hist. Sci. 20 241-76

FitzGerald G 1897 Dissociation of atoms Electrician 21 May 103-4

Hertz H 1883 Experiments on the cathode discharge Ann. Phys., Lpz. 19 782-816

Kaufmann W 1901 The development of the electron idea Electrician 8 November 95-7

Rayleigh 4th Lord 1942 The Life of Sir JJ Thomson (Cambridge: Cambridge University Press; reprinted 1969, London: Dawsons of Pall Mall)

Thomson G P 1964 JJ Thomson and the Cavendish Laboratory in his Day (London: Nelson)

Thomson J J 1897a 'Cathode Rays' Royal Institution Friday Evening Discourse, 30 April 1897, published in The Electrician 21 May 1897 104-9 
_- 1897b Cathode rays Phil. Mag. 44 293-316

------1907 The Corpuscular Theory of Matter (London: Constable)

- 1936 Recollections and Reflections (London: Bell; reprinted 1975, New York: Arno Press) 